\title{
PRESENCIA DE PUBLICACIONES DE MUJERES EN DOS REVISTAS ACADÉMICAS DE HISTORIA EN CHILE ENTRE 1920 Y 1939
}

WOMEN'S PUBLICATIONS IN TWO CHILEAN ACADEMIC MAGAZINES (1920

AND 1939)

Karin Román Guajardo

\section{ReSUMEN:}

El presente artículo se centra en el análisis de publicaciones historiográficas de mujeres chilenas en la Academia de Historia entre 1920 y 1939. Este abarca el contexto histórico que permitió dicha producción académica, ligada al proceso desarrollo de la educación femenina en Chile, el ingreso de las mujeres a la Universidad y la profesionalización del trabajo de éstas, al igual que el estudio de las condiciones materiales e inmateriales de los artículos publicados en las revistas Clío y Revista Chilena de Historia y Geografía. El análisis de los artículos muestra un cambio en los valores atribuidos a las mujeres: Desde un énfasis en temas ligados a la pedagogía, que se desprende del rol tradicional de madre (educadora) en la década del 20, hacia la incorporación de temas propios de la disciplina histórica, que coincidió con la profesionalización femenina durante la década del 30. Asimismo, el impacto de la crisis política de 1925 y económica de 1929 se vio reflejado en la baja del número de publicaciones de mujeres, dejando en evidencia la precaria situación de las mujeres profesionales, abordada por el incipiente movimiento feminista en Chile.

\section{Palabras clave:}

Academia de Historia, educación femenina, revistas académicas, Historia de Chile.

\section{Abstract:}

This article centers on the analysis of women's publications in the Academy of History in Chile, from 1920 to 1939. It describes the historical background that made their work possible: the development of women's education and their access to the University; the professionalization of their work, and the material and unmaterial conditions of the articles written by them and published by Clío and Revista Chilena de Historia y Geografía. The analysis shows a change in societal values attributed to women: Education is the main subject in the 1920's, in relation to the traditional value of motherhood, while during the 1930's they incorporate a variety of topics directly related to the subject of History, which coincides with the professionalization process of women labor. The impact of the political and economic crisis - in 1925 and 1929 respectively- showed in the decline of publications made by women. This reflected the precarious situation of professional women, brought up by the newly established feminist movement in Chile.

\section{KeYWORDS:}

Academy of History, women's education, Academic Magazines, History of Chile. 


\section{INTRODUCCIÓN}

En la actualidad experimentamos un renovado interés por estudiar y valorar las contribuciones de mujeres intelectuales en las distintas ramas de la Academia y las Ciencias Sociales, surgida de un resurgimiento de los movimientos feministas a nivel mundial. Particularmente sobre la Academia de Historia, en el caso chileno nos encontramos con escasas publicaciones que aborden el tema en cuestión, sobre todo en cuanto a la primera mitad del siglo XX. La convergencia entre este renovado interés y la escasez de publicaciones al respecto, nos lleva a dar los primeros pasos en el tratamiento y análisis de las mujeres chilenas en la Academia de Historia, durante la primera mitad del siglo XX.

El ingreso de las mujeres chilenas a la producción del conocimiento científico en sus diversas áreas, se desarrolla al amparo de las universidades. La llegada de las mujeres a la universidad se da gracias a un largo trayecto de perfeccionamiento de la educación femenina en Chile, emprendida desde finales del siglo XIX.

El desarrollo de la educación de las mujeres no se puede desligar de dos procesos fundamentales. El primero es el proceso de fortalecimiento del Estado y adopción de servicios públicos antes desempeñados por la iglesia, enmarcado en la prerrogativa de secularización de la sociedad promovida por los gobiernos liberales. En este puede destacarse el Decreto Amunátegui de 1877, que permite la entrada de las mujeres a la Universidad e impulsa el mejoramiento de la educación secundaria femenina (Sánchez, 2006, p. 498) Si bien el manejo estatal de la educación mejora las condiciones generales de los establecimientos, su cobertura y los currículos educativos, se da una marcada diferencia entre la educación destinada a los hombres y a las mujeres. Hasta 1890 no existían escuelas fiscales para mujeres, por lo que toda su educación recayó en colegios privados o confesionales, y no es hasta 1919 que se estandarizaron los programas educativos femeninos para garantizar una preparación mínima, dirigida a rendir los exámenes de ingreso a la Universidad, en oposición a un currículum colmado por los denominados "saberes femeninos", ligados a la mantención del hogar y cuidado de los hijos (Serrano, 2012, p. 325).

Es por ello que debemos destacar como segundo proceso el paulatino cambio de la idea de los roles de las mujeres en la sociedad, específicamente su participación en el funcionamiento del Estado, que acogió a las primeras profesionales chilenas de diversas áreas, como visitadoras, matronas, enfermeras, médicas y educadoras (Illanes, 2006, pp.19-20). Mientras la idea del rol social del Estado pasa de la caridad al de asistencia, los valores atribuidos a las mujeres y sus capacidades también van transformándose: Desde el ejercicio del cuidado por medio de valores del ámbito doméstico a un ejercicio profesional, colmado de un saber científico propiamente tal (González y Zárate, 2018, 
p. 382). Si bien la concreción de este proceso es de mayor duración que nuestro marco de estudio, nos permite entender las ideas, los roles y valores que enmarcan la labor de las académicas chilenas.

A partir del análisis de dos revistas académicas del período levantamos el problema: ¿Cuál es la tendencia de las publicaciones escritas por mujeres en las revistas académicas Revista chilena de Historia y Geografía y Clío entre el período de 1920 y 1939 ? El territorio de estudio corresponde a Santiago de Chile, lugar de publicación de las revistas, y el período estudiado es de 1920 a 1939.

\section{Hipótesis}

La hipótesis levantada de manera preliminar, plantea que las publicaciones llevadas a cabo por mujeres conlleva dos momentos: Entre 1920 y 1929 están ligadas a las políticas estatales de finales del siglo XIX que impulsan la profesionalización docente femenina, lo que se observa en el cariz pedagógico de las temáticas tocadas por las publicaciones de mujeres en la Revista Chilena de Historia y Geografía. Por otra parte, se evidencia una baja en las publicaciones entre 1930 y 1939, derivada de los efectos de la crisis económica. En paralelo, junto a las mismas políticas estatales, se introduce un cariz académico propiamente tal, con temas distintos del quehacer pedagógico, como se observa en la revista Clío.

\section{Овjetivos}

1. Analizar el contexto de producción de las mujeres relacionadas con las publicaciones académicas de historia.

2. Conocer las condiciones materiales e inmateriales de dicha producción.

\section{Metodología}

Nuestro enfoque parte desde una visión crítica del género, en cuanto a lo que se ha referido el estudio de la integración y la emergencia de la mujer en el mundo educacional, universitario y académico. Consideramos que existe una falta de estudios rigurosos y que logran ponderar los cambios sufridos en su condición de sujeto histórico e intelectual durante el siglo XX.

Es por ello que hemos tratado de integrar una definición y visión de género desde la autora Joan Scott, que se puede dividir en dos partes: por un lado, visto como elemento constitutivo de las relaciones sociales, basadas en diferencias percibidas entre los sexos y, por otro lado, el género como una forma primaria de las relaciones simbólicas de poder. Respecto al primer ámbito, podemos a la vez, subdividir su comprensión 
en cuatro áreas: la primera es, que los símbolos disponibles evocan múltiples y contradictorias representaciones; la segunda es, que los conceptos normativos que existen sobre las interpretaciones de los significados de los símbolos antes mencionados se constituyen básicamente en formas de oposiciones binarias, afirmando de forma categórica e inequívoca el sentido de hombre y de mujer); el tercero, es la restricción que se ha creado en el mundo de las ciencias sociales respecto del uso del mismo término de género, asociándolo al sistema de parentesco, centrado en el hogar y la familia (faltaría integrar su uso en campos como la educación, el mercado de trabajo y el régimen gubernamental); el cuarto, hace referencia al ámbito subjetivo del término, en cómo construye identificaciones a través de las actividades, organizaciones sociales y representaciones histórico-culturales. El segundo eje, referido al género como una forma primaria de desarrollo de las relaciones simbólicas de poder, entiende que los conceptos de género, establecidos como objetivos o conjuntos de referencias, estructuran las percepciones, las organizaciones concretas y simbólicas de la vida social (Scott, 2008. pp. 65-68).

Comprendemos, entonces, que las concepciones de género predominantes en la sociedad científica e historiográfica, son aquellas que han predominado en el análisis de la estructuración del campo intelectual inherente al desarrollo académico de este campo del saber, por lo que una comprensión de los símbolos en su contexto que le han dado forma, nos han permitido entrar a un espacio de análisis bastante inexplorado.

Para el análisis de las revistas, además, y como hemos querido rescatar en los objetivos el ámbito de apropiación es que hemos decidido integrar tres dimensiones de análisis bajo la propuesta de Alexandra Pita y María Grillo. La primera, consiste en el análisis de la dimensión netamente material de las revistas a analizar centrados en su configuración de forma. La segunda, consiste en una dimensión material e inmaterial, centrada en la ponderación de aspectos de contenido, donde se busca la relación entre la forma en que se desarrolla materialmente una revista y el fondo de relaciones sociales e ideas que simbolizan dichas formas de desarrollarlo tanto en su ubicación dentro de la revista como en el tipo de distribución que tenía. La tercera dimensión de análisis que vamos a incorporar es la que corresponde a la inmaterialidad, es decir, todo el ámbito humano en torno a las revistas que nos interesan analizar, por ejemplo, en cuanto a los comités editoriales, las personas que publicaban (grandes escritores, por ejemplo) o quiénes la recibían (González \& Grillo, 2015, pp. 7-24).

\section{Discusión historiográfica: De la PRESENCIA de ESTUdios HISTORIOGRÁFICOS SOBRE MUJERES ACADÉMICAS}


Nos parece importante hacer un punto sobre el estado general de las publicaciones en torno a la temática del rol académico e intelectual de las mujeres en la revisión y análisis de la historiografía chilena.

En términos generales, la mayoría de las obras que hacen una revisión amplia del quehacer historiográfico se han centrado en la coyuntura de reconfiguración intelectual, que se sucedió en la década de 1980 en Chile. No es de extrañar, que en términos cuantitativos y cualitativos, fue una época de emergencia de nuevas historiadoras y cientistas sociales en torno a la publicación de revistas académicas, como también de grupos de estudio extra- académicos.

Desde un enfoque más centrado en la reagrupación intelectual y las propuestas metodológicas y social-políticas, vemos que autores como Gabriel Salazar (Salazar, 2017) y Julio Pinto (Pinto, 2016) han colocado la coyuntura de protestas sociales entre 1983 y 1987 como espacio de eclosión de diferentes grupos intelectuales que contaban con la participación de mujeres historiadoras. Tales fueron los casos del grupo "Encuentro de Historiadores Jóvenes" a partir de noviembre de 1982, con la presencia de historiadoras como Isabel Torres; el grupo de historiadores e historiadoras jóvenes reunidos al alero de la Pontificia Universidad Católica de Santiago, el Instituto Chileno de Estudio Humanísticos, que con un sello socialcristiano reunió a investigadoras como Sol Serrano y Mariana Aylwin; otro caso interesante es la inclusión en el Centro de Estudios Latinoamericanos -cuyo núcleo estaba mayormente compuesto por filósofos centrados en el estudio de la historia de las ideas- de María Angélica Illanes (Salazar, 2017, pp. 37-45).

Colocándose en un plano más temático y teórico, Pinto rescata la inclusión de la categoría de género que habría sido utilizada desde finales de los años ochenta con obras como las de Cecilia Salinas "La mujer proletaria: una historia para contar" de 1987; la obra colectiva del grupo formado por Edda Gaviola, Ximena Jiles, Lorella Lopresti y Claudia Rojas en "Queremos votar en las próximas elecciones: historia del movimiento femenino en Chile" de 1986; como también hace hincapié en el rol de las "chilenistas", especialmente estadounidenses, que desarrollan sus tesis a comienzos de los años noventa; finalmente incluye la importancia de la revista dependiente de la ONG SUR -que venía trabajando desde los años ochenta- en el número 21 de 1992, titulado “Género, Mujer y Sociedad" (Pinto, 2016, pp. 104-105).

Como podemos darnos cuenta, la historiografía se ha centrado en una revisión que abarca mayormente la época más actual en cuanto a la labor intelectual de historiadoras. También, otro tipo de análisis historiográfico existente ha sido el análisis respecto a los estudios de historia de las mujeres como la historia del uso de la categoría de género en Chile. Tales casos son los de María Soledad Zárate y Lorena Godoy (Zárate \& Godoy, 2005), que criticaban la producción historiográfica centrada mayormente en exponer 
la historia de los hombres proletarios como categoría abarcadora de una realidad que excluía a las mujeres. En cuanto a los estudios que han utilizado la categoría de género bajo un balance histórico, a comienzos de los dos mil aparece el artículo de Thomas Klobock "Writing the history of Women and Gender in twentieth-Century Chile" (Klobock, 2001).

Entonces, ¿qué es lo que existe respecto a la historia del campo intelectual de mujeres historiadoras? Contamos con pocos casos de estudios que recobran los orígenes y el desarrollo de dicha actividad, pero existen algunos a mencionar. Primeramente, queremos mencionar la obra en dos tomos de Cristián Gazmuri “La historiografía chilena", que incluye en sus secciones de ensayo bibliográfico la presencia intelectual de mujeres a través de la entrega de algunos datos biográficos y sus escritos. Además, tenemos que colocar acento a que incluye una visión sobre la escritura de la historia bastante más amplia de la que tenemos hoy, respondiendo al contexto de la época y el propio concepto de la historia que abarcaba también géneros como la biografía o la crónica (Gazmuri, 2012). Hablamos del concepto de la historia, puesto que Gazmuri rescató una visión propia de gran parte del siglo XX respecto a la escritura de la historia, expuesta por F. A. Encina en 1935, que le entregaba un concepto bastante ampliado a la idea de "literatura histórica" del siglo XIX, su cultivo extendido y la pérdida de su gran papel a inicios del siglo XX, en detrimento de géneros como la novela, la crítica y la poesía (Encina, 1997, pp. 41-57).

En segundo lugar, dentro de los pocos escritos dedicados a esta temática tenemos que destacar el artículo hecho por Luz María Méndez "La mujer y la historiografía chilena" de 1984, colocando un énfasis importante en la labor inicial a inicios del siglo XX de las mujeres que entraron a la carrera de Pedagogía en Historia y Geografía en el Instituto Pedagógico a cargo de la Universidad de Chile (desde 1890 estuvo a cargo), y que lograron agruparse en torno a publicaciones como "Memorias de los Egresados", la recepción de algunos artículos en la revista Clío -dependiente de la Universidad-, el caso de Amanda Labarca, el de Olga Poblete, el de Elsa Urbina, todas dedicadas a la docencia universitaria desde la década de los treinta hasta mediados de siglo (Méndez, 1984). Otro texto que consideramos sigue esta línea es el que realizaron María Cabrera y Josefina Errázuriz, de 2015, que apuntaba a las publicaciones de mujeres de las revistas Cuadernos de Historia e Historia, que demostraron un avance tanto cuantitativo como cualitativo desde mediados de los años ochenta, siendo parte de la "explosión historiográfica" de los años noventa en el país (Cabrera \& Errázuriz, 2015).

\section{LAS MUJERES Y EL ACCESO A LA EDUCACIÓN Y EL MUNDO DEL TRABAJO EN Chile.}


Para poder comprender cómo se dieron las condiciones de probabilidad para que mujeres pudieran escribir en revista académicas de historiografía en la primera mitad del siglo XX, obligatoriamente nos tiene que remitir a lo que fue el proceso de integración a la educación secundaria y universitaria que vivenciaron como sujeto histórico a finales del siglo XIX.

Con el impulso secularizador que se sufrió en torno a la discusión política y al interior de la elite chilena durante la década de 1870, precedente y base directa de las leyes laicas de 1880, cambió la concepción existente sobre el Estado docente de mediados de siglo. Ésta concebía una educación donde se integraban al hombre y la mujer en roles de género marcados para la época, siendo el primero aquel que tendería a la formación en una profesión, ya fuera técnica o universitaria, y la segunda, recibiría educación que le fuera de utilidad, principalmente, para su función privada al interior de la sociedad, en específico, dentro de la familia. Sin embargo, el cambio de concepción que se sucedió a partir del último tercio del siglo, integró la idea de la mujer como educadora y sostén civilizador principal dentro de la familia, por lo que la discusión por una educación más completa se hizo patente (Serrano, 2012, pp. 320-321)

Autoras como Karin Sánchez destacan la discusión previa que se llevó antes del decreto que permitió la rendición de exámenes libres para la entrada a la universidad de mujeres, el conocido decreto Amunátegui de 1877. Si bien en realidad nunca estuvo prohibido en sí tal derecho, la sociedad chilena, representada en este caso en las acciones del Ministerio de Educación y del Consejo de Educación de la Universidad de Chile, mostraron durante varios años su reticencia y lentitud para integrar peticiones como las de Antonia Tarragó e Isabel Le Brun, ambas directoras de colegios fundados para la educación de mujeres. Estas mujeres directoras de establecimiento educacionales demostrarían un verdadero impulso desde abajo -entendido como la sociedad civil-, que a partir de las peticiones iniciales de Tarragó en 1872 solo tendrían una respuesta hacia 1876, año en que asume el gobierno de Anibal Pinto, y con ello, asume de ministro el mencionado Amunátegui. Lebrun hizo lo propio en 1876, con la petición de acceso a exámenes para sus alumnas y finalmente, después de un traspaso constante de las atribuciones entre el Consejo y el Ministerio, se publica la ley un 5 de febrero de 1877. Bajo la visión de esta autora, dicho decreto de ley debía considerarse como parte del proceso de leyes laicas y secularización liberal del Estado (Sánchez, 2006, pp. 497-514)

Uno de los impulsos principales que permitió la promulgación de la ley fue la posibilidad de incentivar la fundación de Liceos femeninos. Sin embargo, el Estado utilizaría una estrategia conocida que demostró el poco impulso existente desde arriba, puesto que se utilizó la fórmula de subsidio y becas a alumnas, mientras que la conformación de liceos estaría bajo la congregación de padres en sociedades y en base a su propio autofinanciamiento. Sin embargo, sería un impulso autogestionado 
desde las mujeres de la clase alta y media que marcaría un inicio fundamental a partir de los primeros liceos de Valparaíso en 1877, Copiapó en 1878 y Concepción en 1883 (Serrano, 2012, pp. 324-325).

Un aspecto que nos demuestra la importancia de este cambio fue el aumento exponencial de las matrículas femeninas que se dio desde finales del siglo XIX y las primeras décadas del siglo XX. Según Serrano, las estadísticas mostraban que en 1895, las alumnas matriculadas en los liceos fiscales era 110, mientras que para 1908 llegaban a la suma de 5.627 y, para 1927, alcanzaron la suma de 19.580, llegando a aumentar en 187 veces en dicha temporalidad. Sumado a aquello, el porcentaje de asistencia femenina siempre estuvo en un nivel bastante alto y estable, entre el $75 \%$ y el $81 \%$; eso sí, hay que señalar que si bien la cobertura escolar fue en aumento, solo varió entre el 0,9\% en 1907 y el 2,7\% en 1930 (Serrano, 2012, pp. 327-329).

\section{LA ObRa intelectual de MUJeres en las Revistas Clío Y ReVista de la Sociedad Chilena de Historia y Geografía como caso de ANÁlisis: 1920 $-1939$.}

Para entrar en el análisis de las publicaciones de mujeres en las señaladas revistas de historias durante el período de 1920 a 1939, es necesario tener en cuenta la situación de las mujeres como sujetos históricos, y la situación de los colectivos de mujeres desde la sociedad civil. Estos tienen un rol no solo en la reproducción de las políticas estatales ampliamente discutidas, sino que en el cambio de las mismas por medio de discursos que resignifican los valores de la sociedad, provenientes ya no desde la verticalidad de un Estado, sino que desde las mujeres de élite y clase media. Es por ello que nos detendremos brevemente en los discursos de las propias mujeres del período, para observar de qué manera significan su labor dentro del espacio universitario.

En 1927 se cumplen los 50 años del decreto Amunátegui. Como forma de conmemoración un grupo de mujeres santiaguinas que habían concretado sus estudios superiores hasta la fecha conforman un documento denominado "Actividades femeninas en Chile". Entre los distintos escritos, la profesora Sara Guerín realizó un informe sobre los logros de las profesionales chilenas en las diversas áreas del conocimiento a las que ingresaron desde 1877. La obra dice:

\footnotetext{
Aunque personalidades de sobra conocidas, aún más allá de nuestras fronteras, no podríamos prescindir en esta rápida revista a nuestras profesionales, de insertar algunos rasgos de su actuación, a título de ejemplo y para satisfacer el sentimiento de legítimo orgullo que inspiran por sus altas idealidades y por su inquebrantable propósito de con quistar, al lado del hombre, los más valiosos triunfos, primeramente en los estudios y después en el ejercicio de su profesión, cimentando así el prestigio de la intelectualidad femenina. (Guerín, 1928, p. 514)
} 
Si bien Guerín exalta los éxitos de las primeras profesionales universitarias chilenas y su participación destacada en la esfera tradicionalmente masculina, esto no significó despojarse de los valores asociados con la idea tradicional de lo femenino. Si bien la adopción de este nuevo rol por parte de las mujeres de la clase media y la elite correspondió a una ampliación de sus posibilidades de participación en la esfera pública, la narrativa y los valores tradicionales se mantuvieron latentes y condicionantes de gran parte de la trayectoria femenina en la educación superior.

María Aragonés señala que la incorporación de las mujeres a la universidad no se hace pensando en su desarrollo profesional futuro, sino en un incremento cultural y una relativa independencia económica a utilizar en casos especiales, como soltería o viudez $u$ otra dificultad que irrumpa con el esquema de la familia tradicional. A su vez, la mayor incorporación de mujeres a la universidad no implicó una alteración de la imagen del rol femenino tradicional, sino una prolongación de este rol en una formación universitaria que podríamos llamar feminizada, con algunas carreras selectas. Existe por tanto una división de carreras que se corresponden a valores tradicionalmente femeninos con las que son tradicionalmente asociadas con lo masculino (Aragonés, 1978).

La tendencia general de la entrada a la universidad de las mujeres era a las carreras de corta duración y ligadas a las materias subsidiarias de la maternidad y la caridad cristiana, como es el caso de la educación, la salud y el servicio social. Asimismo, el grueso del sistema educativo para 1932 seguía practicando los currículos segregados por género, presentándose los primeros intentos de coeducación en 1932 a pequeña escala y obedeciendo más bien a una falta de recursos que a un esfuerzo por crear un currículum fiscal igualitario. Es por ello que las mujeres llegaban a la universidad con una educación que aún estaba fundamentalmente pensada para las labores domésticas y el cuidado del hogar en lugar de la vista en una trayectoria universitaria como la de los hombres (Austin y Vidal, 2004). Es necesario tener en cuenta que no todos los establecimientos educativos preparaban a las mujeres para rendir exámenes universitarios, y los que lo tenían eran frecuentemente privados, por lo que el acceso era limitado a la clase media y acomodada.

Como contrapunto, las décadas de estudio marcaron también el auge de las primeras movilizaciones feministas en Chile, lideradas por la primera generación de mujeres universitarias, que insta a la crítica de la situación de opresión política, jurídica, económica y social en que se encontraban las mujeres de la época. Entre ellas se destacan el Movimiento pro-Emancipación de la Mujer Chilena (MEMCH) fundado en 1935. La mejora del acceso a la universidad también fue un punto en la agenda política que estas organizaciones persiguieron, además de marcar el comienzo de 
la crítica a los estereotipos de género y el valor de las mujeres solo como esposas y madres, tal como lo expresa su programa (La Mujer Nueva, 3/11/1935).

Si bien la persistencia de la retórica de la profesionalización femenina en torno a valores tradicionales se proyecta con fuerza en el período de estudio, estos mismos valores empezarán a ser puestos en cuestionamiento por parte de las mismas mujeres que lograron acceder a la educación universitaria.

\section{La Academia de Historia y la publicación en Revistas de historia}

En primer lugar, es necesario señalar que el ingreso a la Academia de Historia en el período de análisis está directamente ligado a la carrera de pedagogía, en tanto las Universidades aún no ofrecen las licenciaturas. Como ya señalamos, el estudio de la pedagogía es una de las ramas con más presencia de mujeres en esta época, en tanto la educación se veía como una proyección natural del rol femenino de la madre. Emma Salas plantea que entre 1887 y 1935 las mujeres tituladas de pedagogía correspondían a un $52 \%$ del total de titulados (Salas, 2004).

Sin embargo, es necesario hacer la distinción entre el rubro de la educación y el quehacer académico como tal. Mientras la pedagogía es una de las profesiones que el discurso social adaptó para las mujeres de forma temprana, en tanto se identificaba como la extensión del rol de madre, el quehacer académico está ligado tanto a la producción del conocimiento como a la docencia universitaria, roles que en su naturaleza siguen considerándose un espacio altamente masculino, tal como señala María Ángeles Durán (Durán, 1989). Aquí el acceso de las mujeres se hace más limitado, otro factor que permite explicar la escasa cantidad de publicaciones realizadas por mujeres.

Sobre la disciplina de historia en particular, Luz Méndez establece que las primeras publicaciones de mujeres a principios del siglo XX corresponden a tesis de grado, y se presenta un aumento progresivo de éstas hacia 1926 (Méndez, 1984). Dicho factor lo vemos presente en nuestro análisis de los números correspondientes a la Revista chilena de Historia y Geografía.

Fundada en 1911, por la Sociedad Chilena de Historia y Geografía, contó con la publicación de 95 números entre su período de inicio y 1939. Ilustrativamente, podemos señalar las publicaciones de las tesis de grado para optar al título de profesora de Estado en Historia y Geografía de Edulia Silva Salas "Biografía de Adolfo Ibáñez", aparecida en los números 38 y 39 de 1920 (Revista chilena de Historia y Geografía, 1920); en la publicación de la tesis de Ana Gómez de Latorre "Julio Bañados Espinosa" en los números 53 y 54 de 1924 (Revista chilena de Historia y Geografía, 1924); en este último año, también aparece la publicación de la tesis de grado de Aida Chaparro "El corsario Francisco Drake en Chile" tanto en el número 54 como 55 (Revista chilena de Historia 
y Geografía, 1924, 1925, 1926); y hacia 1927, con la publicación de la tesis de grado de Dora Muñoz “Cartografía primitiva americana” aparecida en los números 59,60 y 61 de 1927 y 1928 (Revista chilena de Historia y Geografía, 1927, 1928). La mayoría de dichas tesis dedicadas al género de la biografía histórica de personajes considerados importantes para la construcción de la historia nacional.

Sin embargo, Méndez advirtió una caída en el número de publicaciones. Las razones para esto no están claras y la autora aventura que puede tratarse de una baja en el interés por publicar o porque las tesis de esos años no lograron la calidad suficiente (Méndez, 1984). En términos cuantitativos, podemos señalar que en la Revista chilena de Historia y Geografía se notó que frente al impulso inicial que se dio entre 1920 y 1925 -donde están la mayoría de artículos de mujeres publicados- con publicaciones en 6 números $(38,39,45,53,54$ y 55), ya se demarcó una clara baja de publicaciones en el quinquenio siguiente, que si bien tuvo una cantidad similar de 6 números con apariciones, tiene que destacarse la ausencia en varios números entre 1926 y 1927 y, una cantidad menor de autoras entre 1927 y 1929, que fueron básicamente la publicación en varios números de la tesis de Dora Muñoz (Revista chilena de Historia y Geografía, 1927, 1928) y de Rebeca Román en los números 64, 65 y 66 de 1929 (Revista chilena de Historia y Geografía, 1929).

Dicha coyuntura es ampliada por Emma Salas en sus razones de una forma más compleja -y en contraste con Méndez-, pues denuncia no solo una caída en las publicaciones de mujeres, sino también una caída en el número de tituladas. La autora señala que entre 1920 y 1928 el número de tituladas aumentó sostenidamente hasta alcanzar un 38\%, cifra que disminuye dramáticamente en 1932 hasta un 15\%, la que repunta nuevamente en 1935 hasta el 38\% (Salas, 2004).

Tanto Salas como Austin y Vidal establecen que este decaimiento podría deberse a factores contextuales más amplios. Ellos ven una relación entre los efectos de la crisis económica mundial, que en Chile golpeó con fuerza en la década del 30, con la caída en las publicaciones de mujeres. Los autores señalan que ellas fueron las más afectadas de la población por la crisis, debido a factores como "prácticas laborales discriminatorias, salarios menores, obstaculización de la defensa de sus derechos laborales y la ceguera para con su doble rol de productora y reproductora de la fuerza laboral." (Austin y Vidal, 2004, p. 60) Esto contribuyó al retiro de muchas mujeres de la universidad, actividad costosa para las familias y que solían privilegiar para el hijo varón.

La revista Clío fue fundada en 1933 por el Centro de Estudiantes de Historia y Geografía de la Universidad de Chile. Entre 1933 y 1937 cuenta con 11 números publicados. 
De 78 publicaciones monográficas entre el período de 1920 y 1939, 10 fueron escritas por mujeres, siendo las autoras Olga Poblete (Revista Clío, 1933), Luisa Frey (Revista Clío, 1933), Elsa Kam-Chings (Revista Clío, 1934), Elena Valle (Revista Clío, 1936), Elizabeth Thiers (Revista Clío, 1936) y Luisa Hucke (Revista Clío, 1937). Entre ellas, solo dos tienen registro actividad académica más allá de una publicación aislada. Estas corresponden a Elena Valle, quien publica 2 artículos con temáticas distintas, y Olga Poblete, quien cuenta con una reconocida trayectoria en la Universidad de Chile y como educadora y activista feminista.

Las temáticas de la revista Clío son variadas y responden a investigaciones de la disciplina histórica y no así del ámbito educativo en diversos ámbitos: Historia Antigua, Historia de oriente, Historia de la Ciencia, Historia Local e Historia Colonial de Chile. Esto constituye una evolución de parte del quehacer histórico de las mujeres de finales del siglo XIX y primeras décadas del XX, en tanto se desligan del género biográfico y hagiográfico para centrarse en temáticas más diversas (Gazmuri).

\section{Conclusión}

En conclusión, consideramos que el decreto Amunátegui de 1877, significó un cambio y ruptura importante dentro de un sistema que mantuvo sus roles de jerarquía y subordinación en cuanto a los roles de género. Esta ruptura estableció una coyuntura crítica a partir del impulso desde abajo de mujeres de sectores altos y medios de la sociedad, que reclamaron por la finalización de la etapa escolar y la entrada al mundo universitario.

La respuesta fue un creciente aumento de la matrícula hacia fines del siglo XIX y las primeras décadas del siglo XX, que permitió establecer la profesionalización de mujeres y su acceso a disciplinas de las que no había sido parte en tiempos pretéritos. En dicho sentido, junto con la profesionalización de disciplinas como la historiografía nacional y la creación de dispositivos culturales como lo fueron las revistas académicas, las mujeres tuvieron una presencia más temprana de lo considerado por los estudios de historiografía que hasta el momento se han desarrollado.

De la mano de la entrada a profesiones como las pedagogías, existió un espacio de desenvolvimiento que sí fue aprovechado y tuvo su presencia en el mundo académico. Esto fue expresado, en un principio desde 1920 a 1930 con la publicación de tesis de pedagogía que abrieron lentamente una serie de temáticas que antes había estado vedadas a éstas como sujeto histórico, y que a la larga, con el paso a la nueva década de 1930 y hasta 1940 significó la diversificación constante de la temáticas tratadas por éstas. 
Es necesario hacer patente que el impulso inicial en las dos revistas estudiadas, tanto la Revista de la sociedad de Historia y Geografía como Clío, fueron dispositivos culturales reflejo de su tiempo, una existencia durante la primera mitad del siglo $\mathrm{XX}$, y no estuvo ajena a coyunturas como las crisis políticas de 1925 o económicas de 1929, por lo que también vemos una caída en la cantidad de publicaciones hechas por mujeres, fenómeno que nosotros aludimos a los efectos que tuvieron dichas crisis sobre nuestro sujeto social.

En adelante queda mucho trabajo por hacer, por lo que presentamos nuestro estudio como un paso a la revisión de un campo intelectual que incluya la perspectiva de género y la presencia de sujetos históricos invisibilizados en su presencia intelectual que reflejaría de mejor forma los cambios al interior de una disciplina como la historiografía y su desarrollo académico.

\section{REFERENCIAS BIBLIOGRÁFICAS}

Aragonés, María (1978). La mujer y los estudios universitarios en Chile: 1957-1974. Covarrubias, P. y Rolando, F. (Comp.) Chile Mujer y Sociedad, Alfabeta.

Austin, Robert y Vidal, Paulina (2004). Género, Intelectuales y Educación Superior en el Siglo XX: La Pared Invisible en Austin, R. (Comp.) Intelectuales y Educación Superior en Chile: de la Independencia a la Democracia Transicional, 1810-2001 (pp. 5996). CESOC.

Cabrera, María Josefina \& Errázuriz, Javiera. (2015). Historia, mujeres y género en Chile: la irrupción de las autoras femeninas en las revistas académicas. Los casos de revista Historia y Cuadernos de Historia. Historia, 1(48), 279-299.

Encina, Francisco Antonio (1997). La literatura histórica chilena y el concepto actual de la historia. Universitaria.

Gazmuri, Cristián (2012). La historiografía chilena (1842-1970) Tomo I y II. Taurus.

Gonzalez, Maricela y Zárate, María Soledad (2018). Profesionales, modernas y carismáticas: enfermeras y visitadoras sociales en la construcción del Estado Asistencial en Chile, 1900-1930. Tempo, 24(2), 369-387.

Illanes, María Angélica (2006). Cuerpo y sangre de la política: la construcción histórica de las visitadoras sociales, Chile, 1887-1940. LOM.

Klobock, Thomas (2001). Writing the history of women and gender in twentiethcentury Chile. Hispanic American Historical Review, 81(1-2), 493-518.

Méndez, Luz María (1984). La mujer y la historiografía chilena. Revista Chilena de Historia y Geografía. (152), 157-178.

Pinto, Julio (2016). La Historiografía chilena durante el siglo XX. América en Movimiento. 
Pita González, Alexandra; Grillo, María (2015). Una propuesta de análisis para el estudio de revistas culturales. Revista Latinoamericana de Metodología de las Ciencias Sociales, 5(1).

Salas, Emma (2004) La Educación Superior y los Movimientos de Emancipación de la Mujer Chilena, 1877-1950. En Austin, R. (Comp.) Intelectuales y Educación Superior en Chile: de la Independencia a la Democracia Transicional, 1810-2001 (pp. 39-58). CESOC.

Salazar, Gabriel (2017). La Historia desde abajo y desde adentro. Taurus.

Sánchez, Karín (2006). El ingreso de la mujer chilena a la Universidad y los cambios en la costumbre por medio de la ley 1871-1877. Historia 2 (39), 497-529.

Scott, Joan (2008). Género e Historia. Universidad Autónoma de la Ciudad de México.

Serrano, Sol; Ponce de León, Macarena \& Rengifo, Francisca (2012). Historia de la Educación en Chile (1810-2010). Tomo II Educación Nacional (1880-1930). Taurus.

Zárate, María Soledad \& Godoy, Lorena (2005). Análisis crítico de los estudios históricos del trabajo femenino en Chile. Centro de Estudios de la Mujer. 